\title{
Transformation of ALK expression and PD-LI 0\% to PD-LI $90 \%$ only after surgery: the need for rebiopsy in lung cancer patients
}

This article was published in the following Dove Medical Press journal: International Medical Case Reports Journal

\author{
Christoforos Kosmidis' \\ Georgios Koimtzis' \\ Dimitrios Giannakidis' \\ Nikos Tteralli' \\ Stylianos Mantalovas' \\ Alexandros Tsakalidis' \\ Konstantina Tsopouridou' \\ Stefanos Atmatzidis' \\ Lazaros Liavas' \\ Paul Zarogoulidis ${ }^{2}$ \\ '3rd Department of Surgery, \\ "AHEPA" University Hospital, \\ Aristotle University of Thessaloniki, \\ Medical School, Thessaloniki, Greece; \\ ${ }^{2}$ Department of Pharmacology and \\ Clinical Pharmacology, School of \\ Medicine, Faculty of Health Sciences, \\ Aristotle University of Thessaloniki, \\ Thessaloniki, Greece
}

Correspondence: Paul Zarogoulidis

Department of Pharmacology and Clinical Pharmacology, School of Medicine, Faculty of Health Sciences, Aristotle University of Thessaloniki, 64 Panorama Street, Thessaloniki, Greece

Tel +306977271974

Email pzarog@hotmail.com

\begin{abstract}
Lung cancer is still diagnosed at a late stage although novel diagnostic techniques are in use. However, as pharmacogenomics have evolved novel targeted therapies either with tyrosine kinase inhibitors or immunotherapy can be currently used as daily treatment. We present the case of a woman with anaplastic lymphoma-positive expression and programmed death-ligand 1 (PD-L1) $0 \%$ score upon diagnosis who underwent therapeutic surgery and represented PD-L1 $90 \%$ expression, however, without anaplastic lymphoma kinase expression. Transformation of the tumor or new tumor is a question to be answered for this patient and possibly we should try and direct rebiopsies for this group of targeted therapy patients.
\end{abstract}

Keywords: lung cancer, adenocarcinoma, EBUS, PD-L1, ALK

\section{Introduction}

Lung cancer is still diagnosed at a late stage although we have novel diagnostic equipment such as the electromagnetic navigation, radial probe endobronchial ultrasound (EBUS), and the convex probe EBUS. ${ }^{1,2}$ However, nowadays the investigation of the tumor genome has led to the development of novel therapies. Non-small-cell lung cancer (NSCLC) patients with metastatic disease and specifically those with adenocarcinoma are being investigated for EGFR, anaplastic lymphoma kinase (ALK), B-Raf proto-oncogene (BRAF), and proto-oncogene tyrosine-protein kinase ROS (ROS1). These genes when expressed in the tumor then tyrosine kinase inhibitors (TKIs) can be administered in oral form. ${ }^{3}$ Recently, programmed death-ligand 1 (PD-L1) was investigated, and the degree of its expression was associated with the effectiveness of immunotherapy, at least for pembrolizumab as first-line treatment. ${ }^{4}$ Currently, there are two immunotherapeutic compounds for second-line treatment: nivolumab and pembrolizumab. Nivolumab has presented effectiveness in second-line treatment indifferent of the PD-L1 expression. ${ }^{5}$ Patients with EGFR-positive expression might develop resistance, and therefore, we investigate either with liquid biopsy or tissue rebiopsy for the T790M mutation. ${ }^{6,7}$ In the case of T790M, osimertinib is administered. We do not need rebiopsy in the case of ALK-positive patient disease relapse, we can administer second-line TKIs directly. There are cases where disease relapse manifests with a different expression, and this is the case that we present below.

\section{Case presentation}

A 65-year-old woman presented with a mass in the left lower lobe $(5 \mathrm{~cm})$ and an enlarged number 11 left lymph node station. Positron emission tomography-computed 
tomography (PET-CT) was performed with positive 9SUV grade from the main lesion and 4.5SUV from the station 11 left; there were no other pathological lymph nodes according to the PET-CT (Figures 1-2). Biopsy was performed from the main lesion with CT guidance, and convex probe EBUS was used for additional staging of the mediastinum (Figure 3). The diagnosis was NSCLC adenocarcinoma from the main lesion; however, the lymph node was negative for cancer. The patient had a body mass index of $40 \mathrm{~kg} / \mathrm{m}^{2}$ and arthritis without any heart problems and a normal spirometry; however; she and her relatives denied surgery and next generation sequencing was performed. Additionally, fluorescence in situ hybridization (FISH) was performed and revealed ALK translocation/fusion (Figure 4). Crizotinib $250 \mathrm{mg}$ was initiated and continued for 2 years without any adverse effects and upon restaging with PET-CT stable disease was observed and the mass upon her final restaging had a diameter of $2.3 \mathrm{~cm}$ and 4SUV and there was no pathologically enlarged lymph node. Therefore, again surgery was proposed and the patient agreed. Sixteen months after surgery, a small lesion of $1.2 \mathrm{~cm}$ was observed right under the diaphragm and next to the vertebra with PET-CT 6SUV and biopsy was performed (Figure 5). Again, it was NSCLC adenocarcinoma with next generation sequencing negative for any gene mutation and negative ALK translocation/fusion with FISH; however, PD-L1 expression was $90 \%$ (Figure 6). We performed PD-L1 in the main tumor lesion and the expression was $0 \%$ (Figure 7).

\section{Staining protocol for PD-LI IHC 22C3 pharmDx Dako}

Step 1: Tissue specimens after deparaffinization and rehydration are led in target retrieval procedure with target retrieval solution, low pH (Code RT100/PT101/ PT200 Dako) for 20 minutes at $66^{\circ} \mathrm{C}$.

Step 2: The Autostainer racks with the specimen slides are placed on Autostainer Link 48 (Dako). The instrument will perform the staining process by applying the appropriate reagent, monitoring the incubation time, and rinsing slides between reagents. The reagent times are preprogrammed in the Dako Link software.

Step 3: Counterstain with hematoxylin (Link) (CodeK8008).

Step 4: Mounting with nonaqueous, permanent media.

Pembrolizumab $200 \mathrm{mg}$ every 20 days was initiated, and the patient is under follow-up till date.

\section{Discussion}

Tumor heterogeneity is well known, and it is observed not only in the main tumor but also between the different

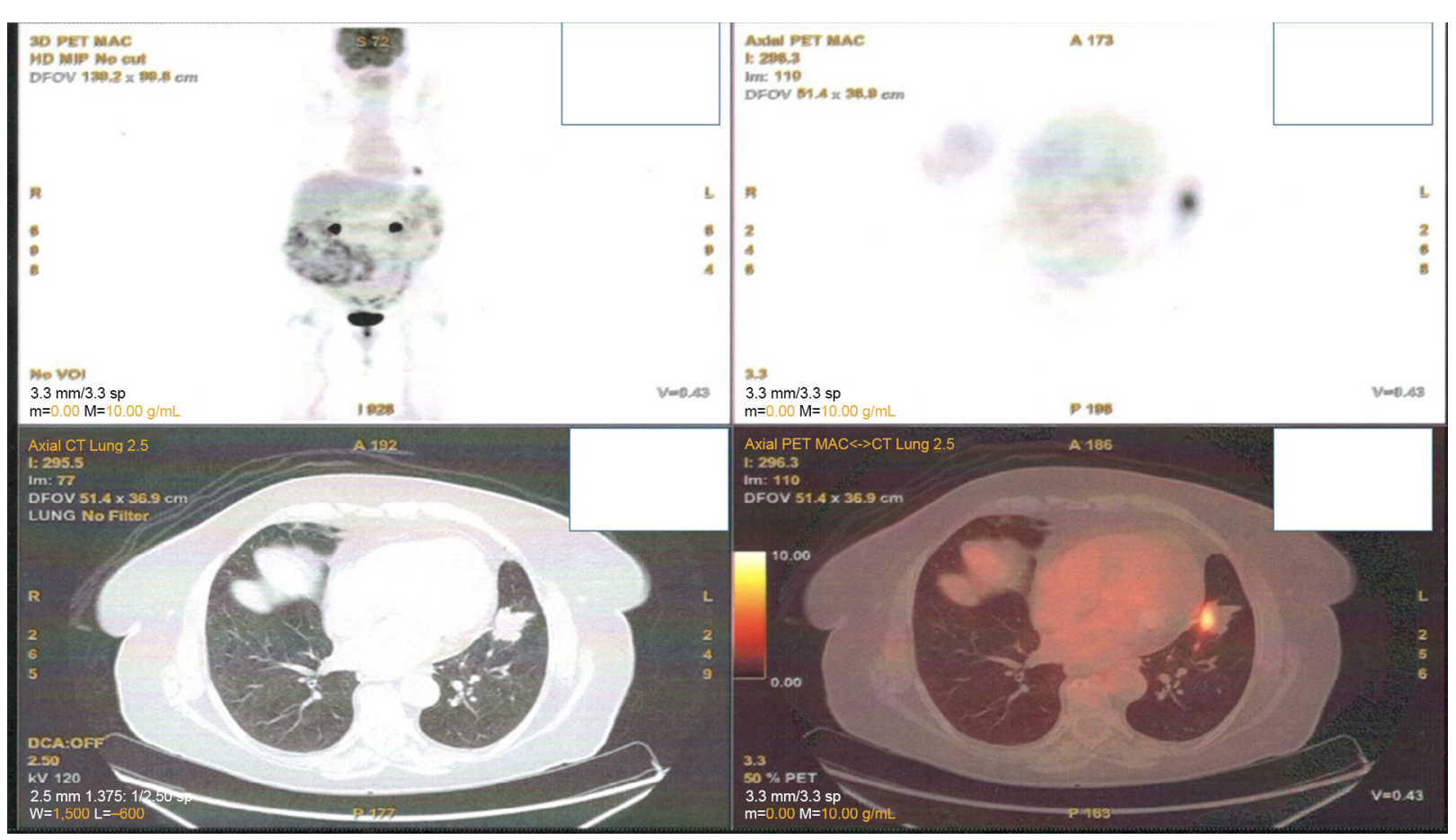

Figure I Main lesion with positive positron emission tomography-computed tomography (PET-CT). 


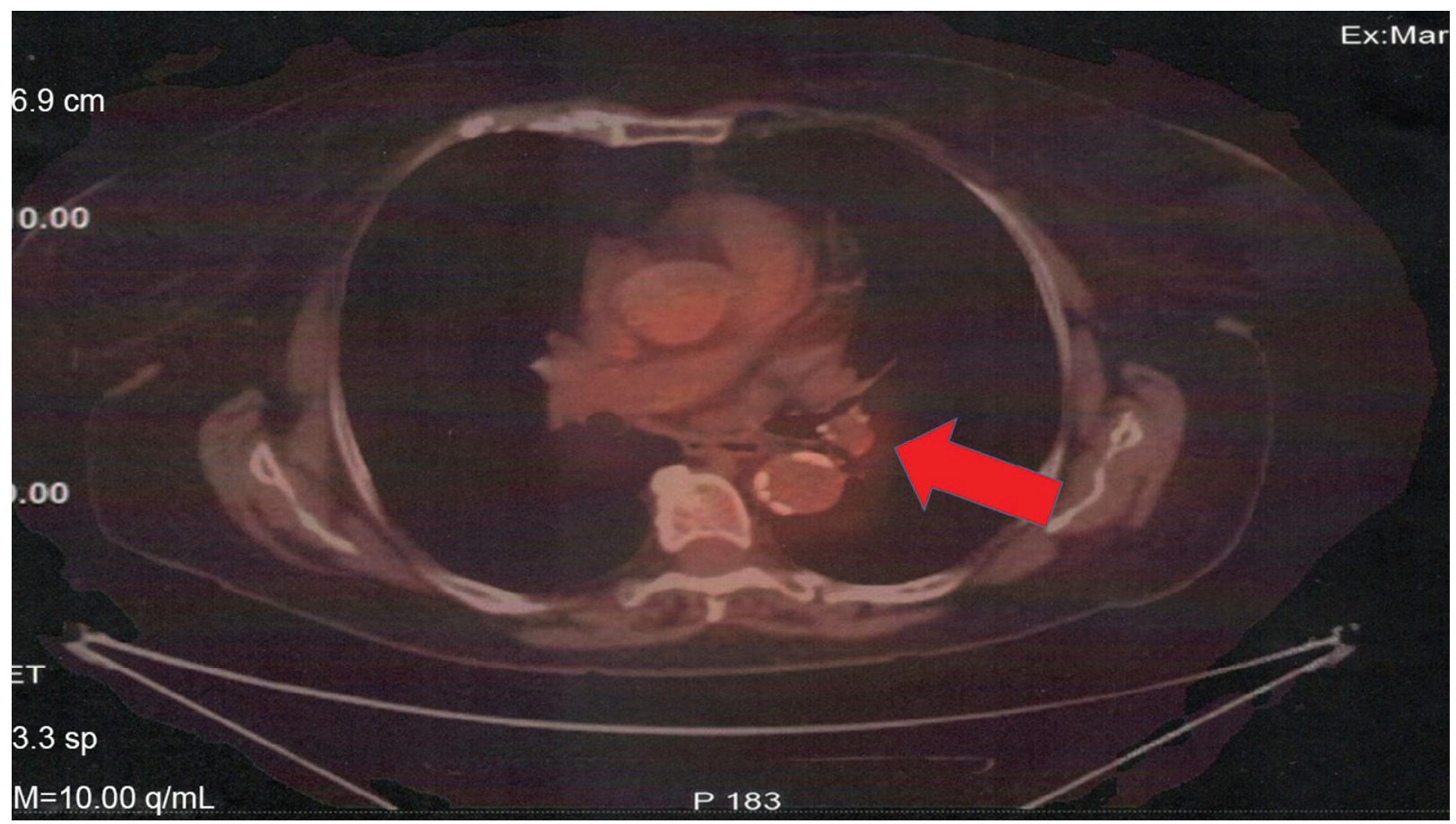

Figure 2 Positron emission tomography-computed tomography with lymph node station number II left (arrow).

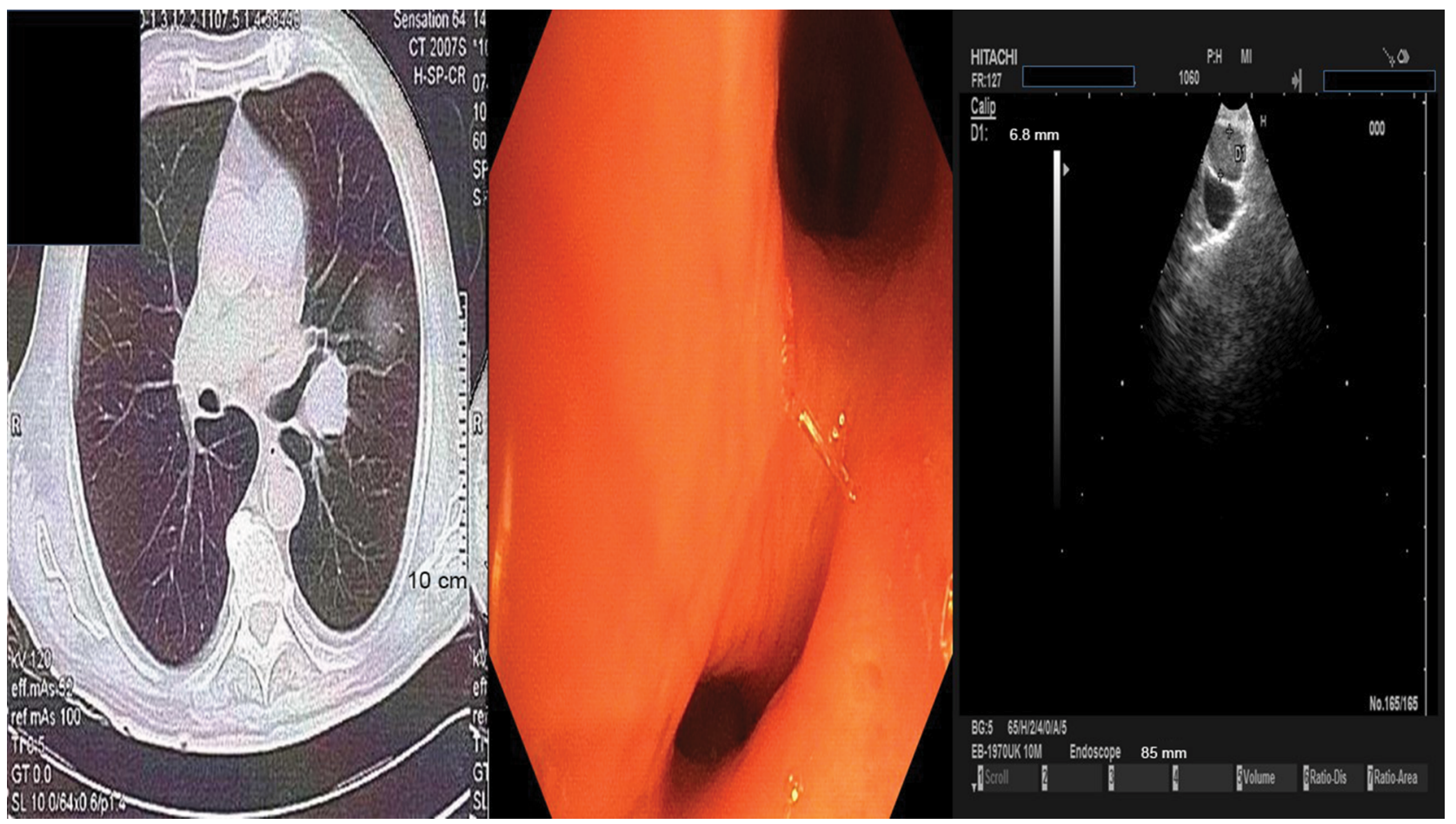

Figure 3 Left, computed tomography of the thorax with lymph node station number II left; middle, the carina of the left upper and lower lobe; and right, the lymph node 22 left with the convex probe ultrasound.

metastatic lesions and lymph nodes. This observation has been verified with surgical specimens. ${ }^{8}$ Moreover, it is known that for several gene expressions such as the ALK,
BRAF, ROS1, and PD-L1, tissue samples are necessary, while for EGFR and T790M cells are enough to determine the gene mutation. ${ }^{9}$ The case that we currently present has 
been observed before in another patient. ${ }^{10}$ Furthermore, it has been previously observed that in the mutation for EGFR and KRAS and translocation/fusion for ALK, these genes play a role in the expression of PD-L1 in the case where the tumor expresses both. ${ }^{11}$ In this case, TKIs should be administered. ${ }^{12}$ It has been observed that a tumor might

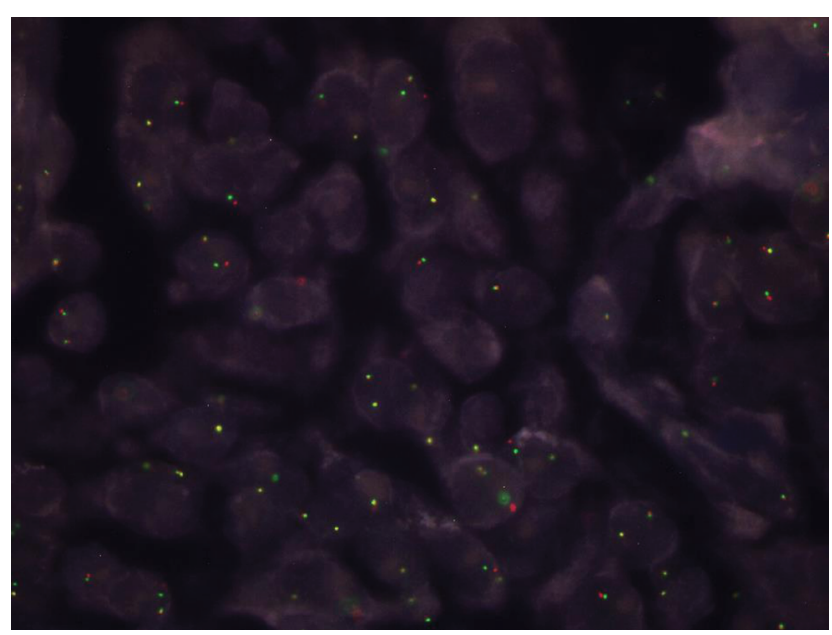

Figure 4 ALK gene rearrangement by FISH in the case of lung adenocarcinoma with positivity in $31 \%$ of neoplastic cells. FISH, ZytoLight SPEC ALK dual color, break apart probe (ZytoVision $\mathrm{GmbH})$.

Abbreviation: ALK, anaplastic lymphoma kinase; FISH, fluorescence in situ hybridization. transform from adenocarcinoma to small-cell lung cancer (SCLC) or the main lesion might remain the same while metastatic sites might transform and then therapy has to change. ${ }^{13,14}$ In another case, an adenocarcinoma with EGFR-positive expression was transformed to SCLC and then again to adenocarcinoma with T790M. ${ }^{15,16}$ Chemotherapy might transform a SCLC to adenocarcinoma with T790M expression, but also, osimertinib might transform an adenocarcinoma with T790M expression to SCLC. ${ }^{15,17}$ There are also cases where afatinib administration transformed adenocarcinoma to $\mathrm{SCLC}^{18}$ and both SCLC and squamous cell carcinoma. ${ }^{19}$ Moreover, it has been observed that ALK-positive patients might transform to SCLC. ${ }^{20}$ Tumor rebiopsy is necessary because tumor transformation might occur or another tumor might arise and appropriate treatment has to be provided.

\section{Ethics approval and consent to participate}

A written informed consent has been provided by the patient to have the case details and any accompanying images published. Institutional approval is not required to publish the case details, only the patients consent.

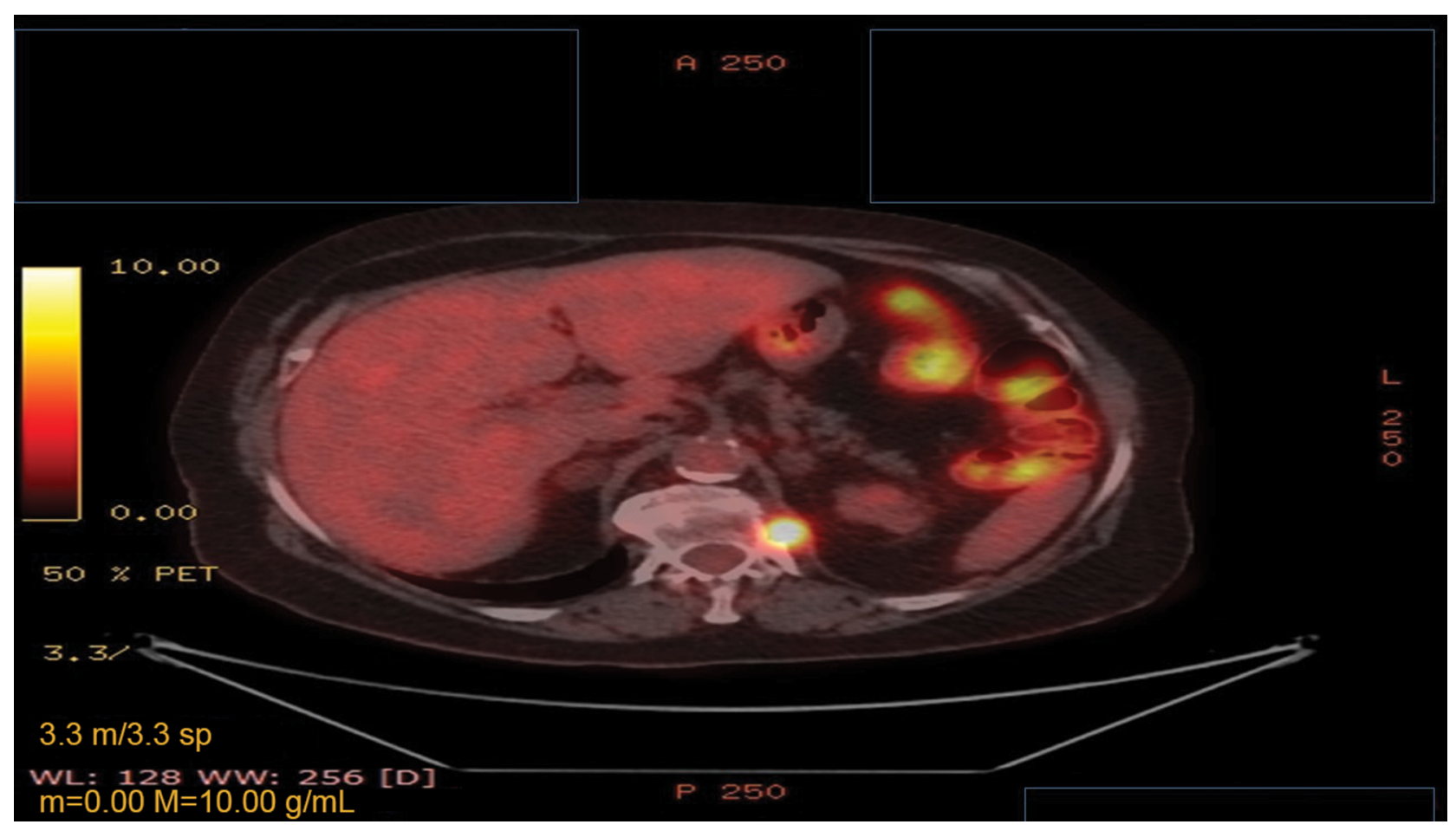

Figure 5 Positron emission tomography-computed tomography with the disease relapse lesion under the diaphragm $1.2 \mathrm{~cm}$. 


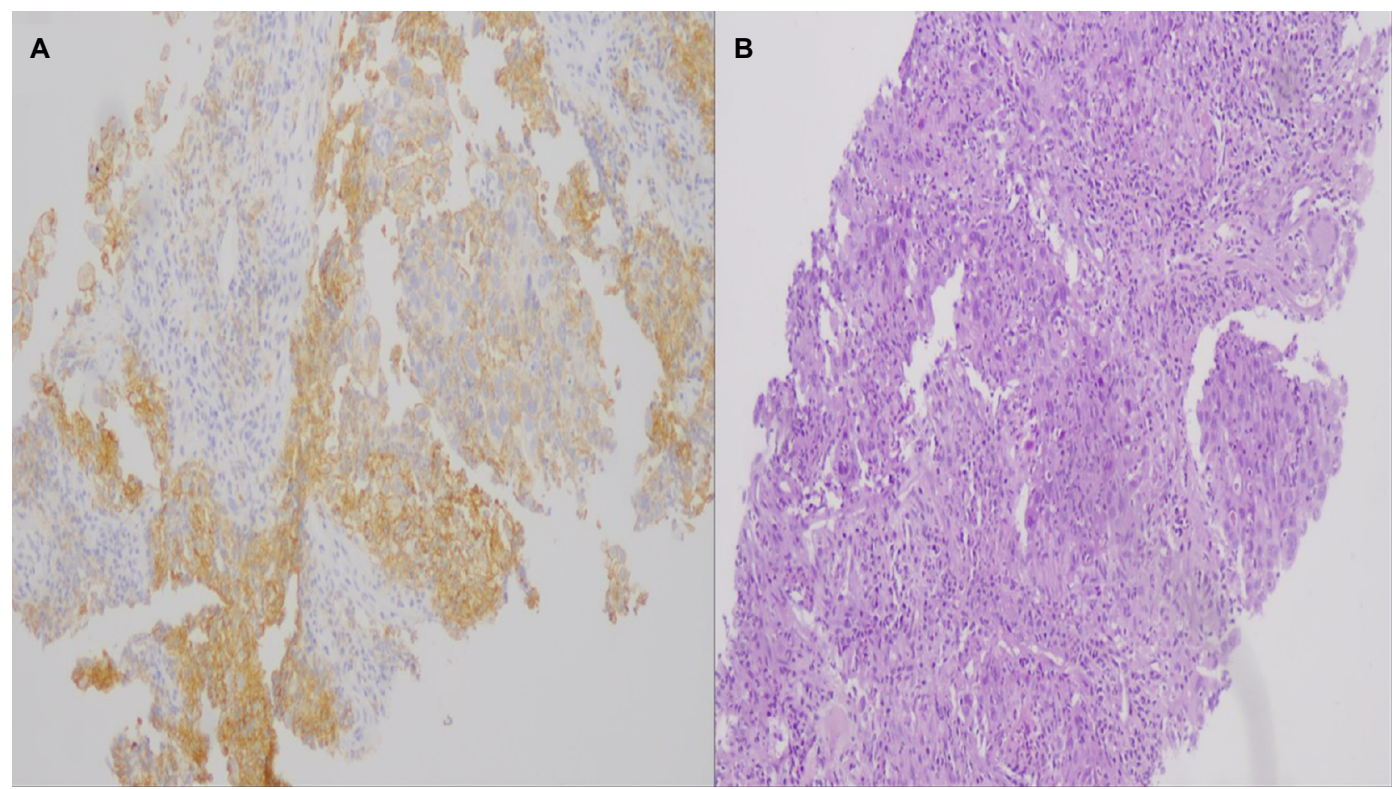

Figure 6 PD-LI expression to the relapse site (nodule under the diaphragm).

Notes: (A) Programmed death-ligand I-positive case with whole membranous staining (intense I+ and 2+) in $90 \%$ of neoplastic cells; (B) hematoxylin/eosin of the same case (undifferentiated lung adenocarcinoma), magnification $\times 100$.

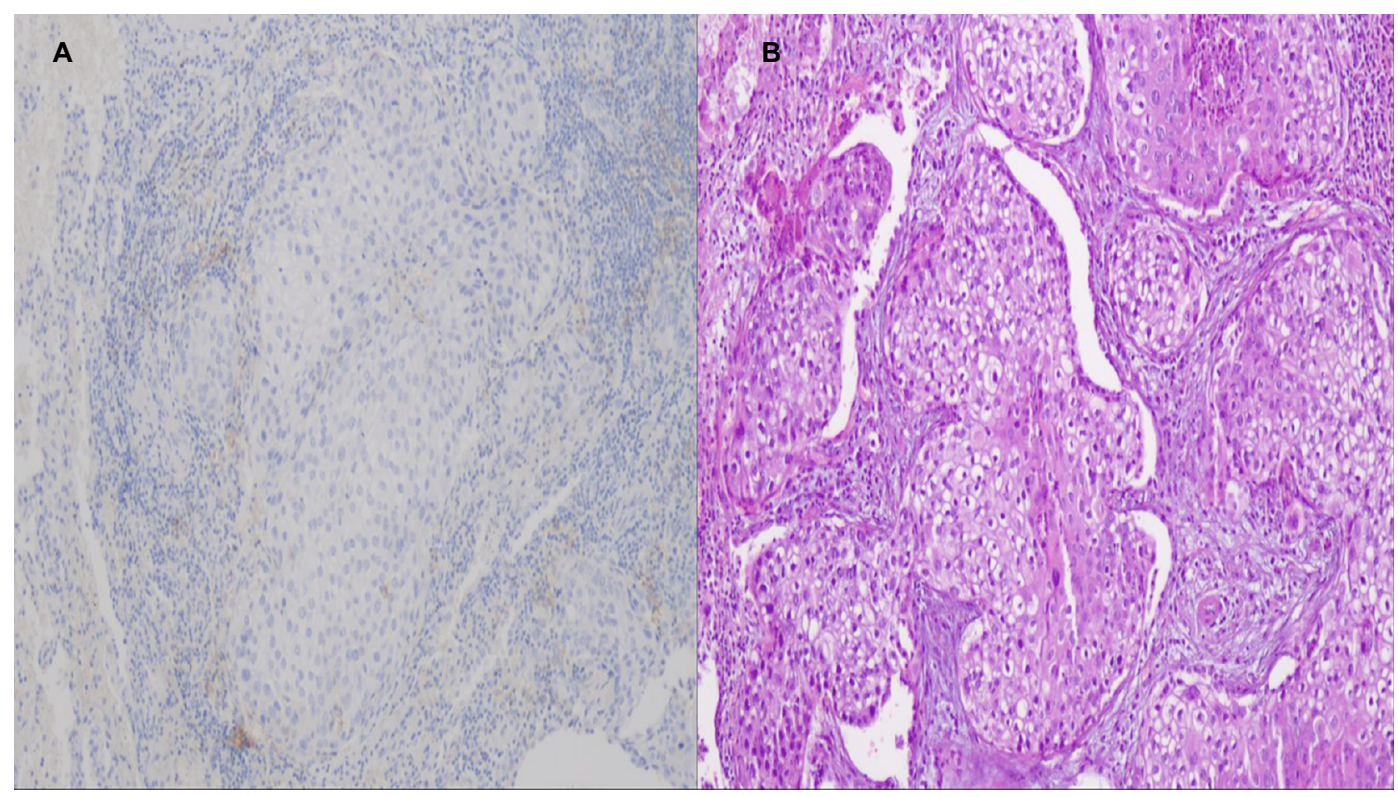

Figure 7 Main tumor PD-LI expression after surgery.

Notes: (A) Programmed death-ligand I-negative case without any membranous, whole or partially positive, magnification $\times 100 ;$ (B) hematoxylin/eosin of the same case with numerous neoplastic adenocarcinoma cells, magnification $\times 100$.

\section{Acknowledgments}

The authors would like to thank Dr Evaggelia Athanasiou and Dr Dimitri Hatzibougia from "Microdiagnostics" private pathology laboratory for their valuable contribution in the diagnosis of the patient. Moreover, the authors would like to thank Dr Dimitri Drouga for his help with the PET-CT diagnosis ("Biomedicine" private scintigraphy laboratory).
Finally, the authors would like to thank the following colleagues for their valuable contribution toward completion of this work: Konstantinos Sapalidis, Nikolaos Michalopoulos, Eleni Georgakoudi, John Koskinas, Maria Florou, Kyriaki Michailidou, Harilaos Koulouris, Isaac Kesisoglou, Maria Kosmidou, Sofia Baka; 3rd Department of Surgery, "AHEPA" University Hospital, Aristotle University of Thessaloniki, 
Medical School, Thessaloniki, Greece; Oncology Department, "Interbalkan" European Medical Center, Thessaloniki, Greece.

\section{Disclosure}

The authors report no conflicts of interest in this work.

\section{References}

1. Zaric B, Stojsic V, Carapic V, et al. Radial endobronchial ultrasound (EBUS) guided suction catheter-biopsy in histological diagnosis of peripheral pulmonary lesions. J Cancer. 2016;7(1):7-13.

2. Zarogoulidis P, Huang H, Bai C, et al. Endobronchial ultrasound convex probe for lymphoma, sarcoidosis, lung cancer and other thoracic entities. A case series. Respir Med Case Rep. 2017;22:187-196.

3. Tsoulos N, Papadopoulou E, Metaxa-Mariatou V, et al. Tumor molecular profiling of NSCLC patients using next generation sequencing. Oncol Rep. 2017;38(6):3419-3429.

4. Cetin B, Bilgetekin İ, Ozet A. Current uses of check inhibitors in the fight against advanced and/or metastatic lung cancer: will immunotherapy overcome chemotherapy? Curr Probl Cancer. Epub 2018 Aug 4.

5. Rounds A, Kolesar J. Nivolumab for second-line treatment of metastatic squamous non-small-cell lung cancer. Am J Health Syst Pharm. 2015;72(21):1851-1855.

6. Zarogoulidis P, Rapti A, Sardeli C, et al. Re-biopsy after relapse of targeted therapy. T790M after epidermal growth factor mutation, where and why based on a case series. Respir Med Case Rep. 2017;21:171-175.

7. Dahl E, Kloten V. [Liquid biopsy analysis using cell-free DNA (cfDNA): opportunities and limitations]. Pathologe. 2015;36(6):572-578.

8. Wu P, Wu D, Li L, Chai Y, Huang J. PD-L1 and survival in solid tumors: a meta-analysis. PLoS One. 2015;10(6): 0131403.

9. Heitzer E, Ulz P, Geigl JB. Circulating tumor DNA as a liquid biopsy for cancer. Clin Chem. 2015;61(1):112-123.
10. Shimada M, Tamura A, Yokosuka K, et al. A successful pembrolizumab treatment case of lung adenocarcinoma after becoming resistant to ALK-TKI treatment due to G1202R mutation. Respir Investig. 2018;56(4):365-368.

11. Bassanelli M, Sioletic S, Martini M, et al. Heterogeneity of PD-L1 expression and relationship with biology of NSCLC. Anticancer Res. 2018;38(7):3789-3796.

12. Zarogoulidis P, Chinelis P, Efthymiou C, et al. EGFR or PD-L1 decision for first line therapy in a case series of EGFR positive and PD-L1 $>50$. Respir Med Case Rep. 2017;22:7-10.

13. Oser MG, Niederst MJ, Sequist LV, Engelman JA. Transformation from non-small-cell lung cancer to small-cell lung cancer: molecular drivers and cells of origin. Lancet Oncol. 2015;16(4):e165-e172.

14. Chen B, Hu B, Li W, Xue J. Transformation from NSCLC to SCLC: when did it happen? Lancet Oncol. 2015;16(7):e309.

15. Sonoda T, Nishikawa S, Sakakibara R, et al. EGFR T790M mutation after chemotherapy for small cell lung cancer transformation of EGFR-positive non-small cell lung cancer. Respir Med Case Rep. 2018;24:19-21.

16. Lee S, Joo J, Kwak M, Sohn K, Chon S. Role of chemotherapy with epidermal growth factor receptor-tyrosine kinase inhibitor (EGFR-TKI) rechallenge in small cell transformation after EGFR-TKI failure: a case report. Onco Targets Ther. 2018;11:3943-3947.

17. Taniguchi Y, Horiuchi H, Morikawa T, Usui K. Small-cell carcinoma transformation of pulmonary adenocarcinoma after osimertinib treatment: a case report. Case Rep Oncol. 2018;11(2):323-329.

18. Shiroyama T, Nasu S, Tanaka A, et al. Transformation to small cell lung cancer after first-line afatinib treatment. Respir Med Case Rep. 2018;23:188-190.

19. Yao Y, Zhu Z, Wu Y, Chai Y. Histologic transformation from adenocarcinoma to both small cell lung cancer and squamous cell carcinoma after treatment with gefitinib: a case report. Medicine. 2018;97(18):e0650.

20. Hobeika C, Rached G, Eid R, et al. ALK-rearranged adenocarcinoma transformed to small-cell lung cancer: a new entity with specific prognosis and treatment? Per Med. 2018;15(2):111-115.
International Medical Case Reports Journal

\section{Publish your work in this journal}

The International Medical Case Reports Journal is an international, peer-reviewed open-access journal publishing original case reports from all medical specialties. Previously unpublished medical posters are also accepted relating to any area of clinical or preclinical science. Submissions should not normally exceed 2,000 words or

\section{Dovepress}

4 published pages including figures, diagrams and references. The manuscript management system is completely online and includes a very quick and fair peer-review system, which is all easy to use. Visit $\mathrm{http}: / /$ www.dovepress.com/testimonials.php to read real quotes from published authors. 